This item was submitted to Loughborough's Research Repository by the author.

Items in Figshare are protected by copyright, with all rights reserved, unless otherwise indicated.

\title{
Tracking multiple mobile targets based on the ZigBee standard
}

PLEASE CITE THE PUBLISHED VERSION

PUBLISHER

(C) IEEE

VERSION

VoR (Version of Record)

LICENCE

CC BY-NC-ND 4.0

REPOSITORY RECORD

Alhmiedat, Tareq Ali, and Shuang-Hua Yang. 2019. "Tracking Multiple Mobile Targets Based on the Zigbee Standard”. figshare. https://hdl.handle.net/2134/5990. 
This item was submitted to Loughborough's Institutional Repository (https://dspace.lboro.ac.uk/) by the author and is made available under the following Creative Commons Licence conditions.

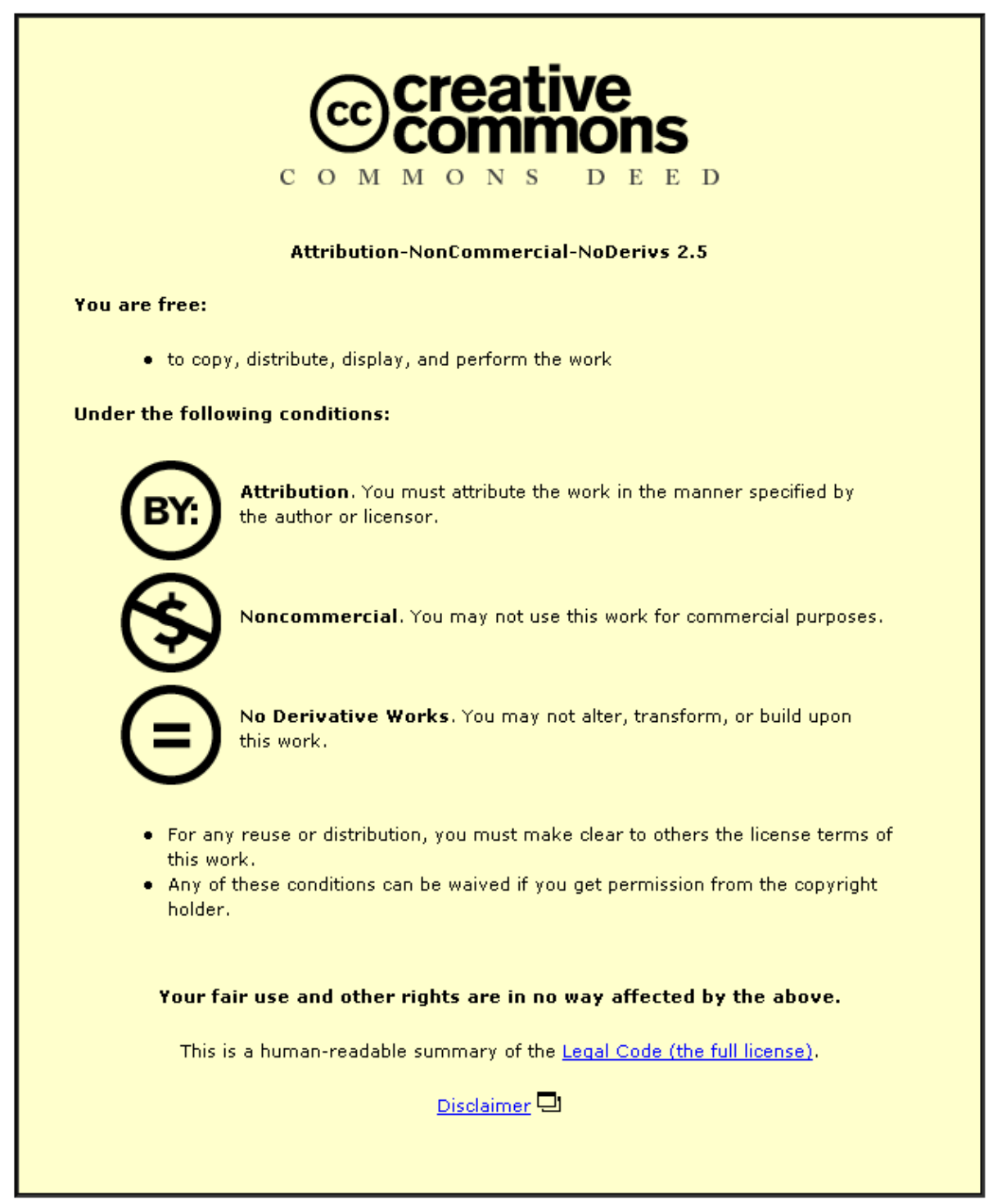

For the full text of this licence, please go to: http://creativecommons.org/licenses/by-nc-nd/2.5/ 


\title{
Tracking Multiple Mobile Targets Based on the ZigBee Standard
}

\author{
Tareq Ali Alhmiedat, Shuang-Hua Yang \\ Computer Science Department, Loughborough University \\ (T.Alhmiedat, S.H.Yang)@lboro.ac.uk
}

\begin{abstract}
Target localization and tracking problems in Wireless Sensor Networks (WSNs) have received considerable attention recently, driven by the necessity to achieve a higher localization accuracy, a lower cost, and a smallest form factor. Despite the interest on target tracking and localization issues, there are few systems deployed using ZigBee network standard, and there is no tracking system that has used the full roles for ZigBee network standard. Tracking through the ZigBee network standard is a challenging task when the density of router and end-device nodes is low, due to the limited communication capabilities of end-device nodes. This paper addresses the research challenges in localization and tracking of multiple mobile targets through the implementation of a ZigBee based real-time surveillance applications. The proposed system is a power-efficient system that does not require any additional hardware devices, and can be deployed where the density of beacon nodes is low. This proposed tracking system can be applied in various indoor applications, such as military, civil, and health applications.
\end{abstract}

\section{INTRODUCTION}

Wireless Sensor Networks (WSNs) have become a common research area, primarily due to their wide-ranging applications. A WSN consists of spatially scattered autonomous sensor devices that cooperatively watch physical or environmental conditions such as humidity, temperature, pressure, sound, or motion of objects at different locations. Sensor networks are comprised of sensor nodes which are very small in size, low in cost, and a low range communication network.

The node localization and tracking applications through WSN have received much attention recently, focused on the need to achieve high localization accuracy without incurring a large cost, form factor and power-consumption per node. This is because:

1. In several applications, the location itself is the information of interest.

2. Transferring a sensor's measurements without knowing its position provides useless information.

3. Several routing protocols are based on knowing the locations of sensor nodes.

Practically, designing and developing a localization method for WSNs is a complicated task due to the limited capabilities of sensor devices. Tracking using a ZigBee network has received little attention due to the limited communications capabilities of such a network. ZigBee is a new emerging technology, which has been released recently for several applications such as home automation, healthcare, security, and safety applications. The research challenge is thus, how to obtain high localization accuracy and track multiple mobile targets using a ZigBee WSN in complex environments, at the lowest possible cost.

There are several localization technologies have been used for diverse applications, such as infrared, ultrasound, and Global Positioning System (GPS). These solutions offer high localization accuracy but suffer from different problems, such as cost and size factors. In addition, GPS and infrared technologies are not applicable solutions for indoor applications. Received Signal Strength (RSS) localization systems are among the most popular and cheapest techniques, and offer reasonable localization accuracy outdoors, but are effected by obstacles and walls in indoor environments.

The proposed tracking system in this paper is based on a RF localization method. However, the interference issue can affect the localization system and hence offers low accurate localization estimation. A time management method is proposed in this paper in order to overcome the interference limitation through localizing target nodes in WSN.

The main contributions of this paper are the following: a) a new tracking approach is proposed to localize multiple mobile targets practically using ZigBee; b) the proposed ZigBee-based tracking approach reduces the amount of energy consumed when a high density of end-device nodes are involved in the tracking process; c) the presented system achieves low localization error in a complex environment with obstructions where the density of beacon nodes is low; d) finally, a low signal interference rate is achieved between beacon nodes, which significantly reduces the localization error.

This paper is divided as follows. In Section 2, the current localization and tracking systems are listed. A new localization system based on ZigBee is proposed in Section 3. Section 4 reviews the implementing proposed system on real ZigBee-based sensor devices. Section 5 provides an evaluation of the proposed tracking system. A discussion is presented in Section 6. And finally, Section 7 concludes the proposed system and presents future works.

\section{BACKGROUND}

Tracking is the ability to find out the location of target nodes in real time. Usually, each tracking system consists of one or more localization technique. Localization techniques are based on some form of communications between beacon nodes with known positions and target nodes which need to 
be localized. In this section, we present several localization systems.

\section{A. Related Work}

The issue of tracking mobile targets through WSN is actively researched and addressed in several works. A survey on localization and tracking system through WSN is presented in [1].

In this section, the related works are divided into two main categories based on the deployed network standard: localization systems based on other network standards, and localization systems based on the ZigBee network standard.

Firstly we consider localization systems using other network standards. The proposed localization systems in [2, $3,4,5]$ offer efficient localization information, but they cannot be deployed using ZigBee standard.

Secondly, several ZigBee-based localization systems have been proposed recently [6, 7, 8, 9]. Each one of these systems deploys different localization method, even though all of these systems were deployed using ZigBee standard.

Several ZigBee-based localization systems were deployed based on router and coordinator network components $[8,10$, 11]. However, end-device nodes were not used in these experiments due to their limited communication capabilities. Power-consumption is a critical issue in WSN applications, as each sensor device has limited battery power. End-device nodes can reduce the power-consumption for the whole WSN as they can go into sleep mode when they have completed their tasks. Little work has so far been done in tracking based on the ZigBee specification. A ZigBee network with a high density of end-device nodes offers a low-power-consumption and efficient sensor network. However, it also offers low localization accuracy when multiple mobile nodes need to be tracked.

In this paper, we address the gaps for the previously presented localization systems including localization accuracy, interference, and tracking multiple objects using the ZigBee.

\section{TRACKING MULTIPLE MOBILE TARGETS APPROACH}

In this section, we briefly describe the ZigBee network standard, discuss the limitations of deploying a RSS-based localization system using ZigBee standard, and propose a solution for tracking multiple mobile targets.

\section{A. ZigBee Network Standard}

Several network standards have been released recently such as 802.11a, b, g, Bluetooth, 802.15.4, and ZigBee. The presented tracking system is deployed through the ZigBee network standard.

ZigBee is a low data rate, low power, and low cost wireless communication standard which is designed for the use in home automation and remote control applications. The ZigBee network includes three main device types as shown in Figure 1: coordinator, router, and end-device.

The coordinator has the roles of starting and controlling the network in addition to storing information about the whole network. The router is responsible for routing messages between nodes, and providing backup routes in case of network congestion or device failure. Each router can communicate to a coordinator, and other routers in its range, and end-device nodes. End-device has the ability to transfer and receive messages, but cannot perform any routing operations. Each end-device can only connect to a coordinator or a router, and cannot have child devices.

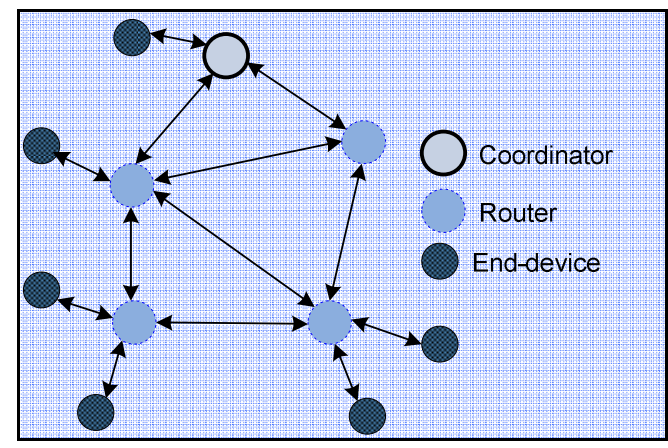

Figure 1. ZigBee Network Standard

The most important advantages behind deploying our tracking system using the ZigBee network standard is the simplified implementation process with the provided protocol suit of ZigBee. Furthermore, the low complexity, fast calculation, and minimum resource requirements, make ZigBee standard an ideal network solution for wireless sensor nodes.

\section{B. Tracking Limitations Using ZigBee}

Localization methods based on RSS technique require a minimum of three beacon nodes to triangulate a mobile target's position. Consequently, due to the previously discussed characteristics of end-devices, where each enddevice can only communicate with one parent node, there may not be three beacon nodes available for triangulation of the mobile target node.

In a ZigBee WSN this can be caused by one of two issues:

1. The existing end-devices are already connected to existing routers in the WSN. Hence this mobile node can only communicate with the router nodes.

2. In systems where multiple mobile targets that need to be tracked. End-devices in a given area may already be connected to a mobile target. Consequently, a second mobile node moving into the given area will not be able to connect with the end-devices until the first mobile node is disconnected.

A possible solution to the problem for ZigBee networks, when the problem arises due to issue 1 is proposed in [12]. The solution presented involves disconnecting the enddevices from the previous router node and reconnecting them to the mobile target. This system is applicable for tracking a single mobile target. However it is not applicable for multiple mobile targets tracking. A practical solution is presented in the next section to solve this second issue.

\section{General Tracking Process}

For simplicity, we break up the tracking process into five phases. Table 1 shows several parameters definition. 


\section{Detection}

Detecting the first position of the mobile target can help in reducing the localization error. This process can be achieved by fixing router nodes at each entrance in the tracking area. As soon as one of them receives signals from any target node, it reports this to the coordinator and all beacon nodes in its vicinity.

TABLE I

PARAMETERS DEFINITION

\begin{tabular}{|l|l|}
\hline \multicolumn{1}{|c|}{ Parameter } & \multicolumn{1}{c|}{ Definition } \\
\hline$b_{i}$ & beacon node with serial number $i$ \\
\hline$b r_{i}$ & beacon router node with serial number $i$ \\
\hline$b e_{i}$ & beacon end-device node with serial number $i$ \\
\hline$M T_{j}$ & $\begin{array}{l}\text { Mobile target node with serial number } j \\
\text { nodes } b_{i} \text { and } b_{j}\end{array}$ \\
\hline$R S S\left(b_{i}, b_{j}\right)$ & $\begin{array}{l}\text { the separation distance between a beacon node } \\
b_{i} \text { and mobile node } M T_{k}\end{array}$ \\
\hline$d i s t\left(b_{i}, M T_{k}\right)$ & $\begin{array}{l}\text { environment factor for two adjacent beacon } \\
\text { nodes } b_{i} \text { and } b_{j}\end{array}$ \\
\hline$e f_{i j}$ & $\begin{array}{l}\text { the average environment factor between three } \\
\text { beacon nodes, where they form a triangle }\end{array}$ \\
\hline$\mu_{e f}$
\end{tabular}

\section{Search}

During the search stage, the mobile target tries to find three beacon nodes to which it can connect to triangulate its position. This consists of three stages:

1. The mobile target node tries to find three beacon router nodes to connect with initially, because they give the highest localization accuracy. If less than three router nodes are available, the mobile target node connects with any available routers if any, and then starts stage 2 .

2. The mobile target node searches for available beacon end-devices in range and connects with enough enddevices, so that the total number of beacon routers $n_{r}$ and end-devices $n_{e}$ connected to the mobile target equal three. As shown in Figure 2, the mobile targets $M T_{1}$ and $M T_{2}$ are connected to $\left\{b r_{1}, b e_{3}, b e_{4}\right\}$ and $\left\{b r_{1}, b e_{6}, b e_{7}\right\}$ respectively. However, if there are not enough end-devices to satisfy (1), as the mobile target $M T_{3}$ can only connect to a single router node, then the mobile target enters stage 3 .

$$
n_{r}+n_{e}=3
$$

3. The mobile target node searches for other mobile targets in range, so that the total number of routers $n_{r}$, end-devices $n_{e}$, and other mobile targets $n_{m}$ connected to the mobile target are equal to 3 . However, if there are not enough mobile targets in range, then triangulation of the mobile node is not possible.

$$
n_{r}+n_{e}+n_{m}=3
$$

However, tracking multiple mobile target nodes can be carried out by dividing the multiple target models into a series of single mobile target nodes, in order to solve the previous problem.

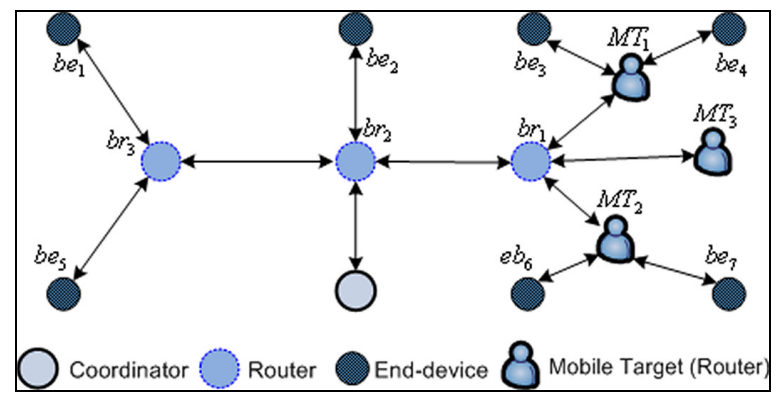

Figure 2. Tracking limitation through ZigBee standard

\section{Selection}

ZigBee Network layer (NWK) provides the mechanisms for joining and leaving the network, applies security to frames, and routes messages to their intended destinations. The discovery of one-hop neighbors and saving of relevant neighbor information are carried out at the NWK layer. Each mobile target node keeps a routing table, which includes information about neighboring nodes in order to transmit messages to them.

After detecting the first position for the target node, the target node has to select 3 beacon nodes in its range in order to triangulate its current location. Through our experiments, it has been noticed that the indoors transmission range for a Jennic sensor node is 20-25 meters (m). Furthermore, it has been found that localizing a target node based on 3 beacon nodes offers efficient localization accuracy, compared with localization based on more than 3 beacon nodes. The difficulty in calculating the localization error increases as the number of environment factors increases. Moreover, using a large number of environment factors causes the WSNs energy levels to be depleted faster. However, it is possible for a mobile target to connect with more than 3 beacon nodes. This results in the problem of which 3 router nodes to choose to use for triangulation.

A RSS-based localization system is proposed in [13] to reduce the positioning error for localizing a single target node based on the ZigBee network standard in indoor environment. This system works by finding out the relationship between RSS values and distances for each pair of beacon nodes in order to predict the environment characteristics. The calculated environment factor ef (presented in Equation 3) between beacon nodes can offer some prediction information to a mobile target node in order to reduce the localization error. The proposed system was implemented with a small number of beacon nodes ( 3 beacon nodes). In this paper, we expand the method to make it reliable when used with a high number of nodes.

There are 4 different triangles $\left(\Delta a_{1}, \Delta a_{2}, \Delta a_{3}, \Delta a_{4}\right)$ which can be formed as shown in the tracking area in Figure 3. Each mobile target $M T_{i}$ is covered by total number of beacon 
nodes $n$. The average environment factor $\mu_{\text {ef }}$ is calculated between each 3 adjacent beacon nodes whên they form a triangle shape, as presented in Equation 4. For instance, in Figure 3, if a mobile target $M T_{i}$ can talk to these beacon nodes $\left(b_{1}, b_{2}, b_{4}, b_{5}\right)$ there will be two possible triangles $\Delta a_{1}$ and $\Delta a_{2}$ which consist of $\left(b_{1}, b_{4}, b_{5}\right)$ and $\left(b_{1}, b_{5}, b_{2}\right)$ respectively. Equation 5 has to be applied in order to find the right triangle which includes the target node.

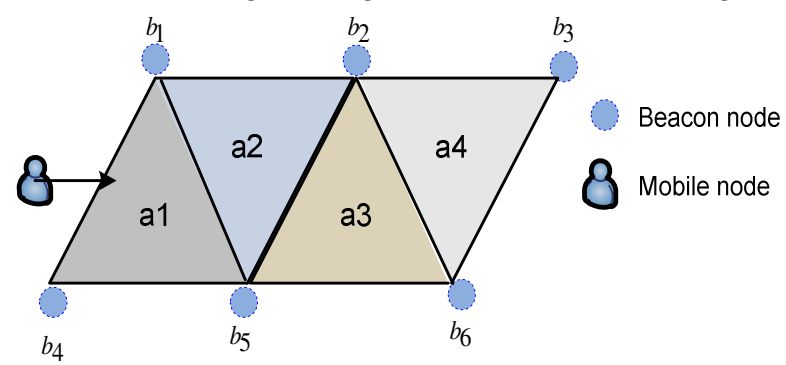

Figure 3. Localization through area of triangles

$$
\begin{array}{r}
e f_{i j}=\left(\frac{r s s\left(b_{i}, b_{j}\right)}{\operatorname{dist}\left(b_{i}, b_{j}\right)}\right) \\
\mu_{e f}=\frac{\sum_{i=1}^{3} e f_{i j}}{3} \\
\operatorname{dist}\left(b_{i}, m_{k}\right)=\frac{r s s\left(b_{i}, m_{k}\right)}{\mu}
\end{array}
$$

\section{Localization and Tracking}

In this phase, we discuss two issues: interference and localization. First, interference affects coverage and capacity, and limits the effectiveness for the localization system. In this section, we focus on the interference among ZigBeesensor nodes.

Through our experiments, we have found that transmitting packets from 2 beacon nodes to one mobile target at the same time can apparently increase the localization error, as sometimes both of the transmitting nodes use the same channel.

There are two methods to overcome the interference between sensor nodes. The first one is the frequency diversity which means that each message has to be transmitted separately at two different frequencies. The work presented in [10] includes an indoor localization system based on a RSS system. The proposed system was tested using Chipcon module which feature a simple frequency switching between $868.28 \mathrm{MHz}$ and $915.03 \mathrm{MHz}$. Transmitting each message in two different frequencies from all beacon nodes to each mobile target adds additional cost and powerconsumption.

The second method includes transmitting messages to the mobile node in several time slots in order to avoid collision between adjacent beacon nodes. For instance, if the mobile target is covered by 3 beacon nodes, then the mobile target will manage to communicate to each beacon node at each time. This reduces the interference between beacon nodes and consequently improves the localization accuracy. We have deployed this method in our proposed system, in order to reduce the localization error at the lowest cost possible.

Secondly, the localization method. RSS-based localization systems have been deployed widely in several works.

The selection process requires finding out the suitable triangle which includes the target node. Once the correct area has been identified, the localization method in [12] is used to further reduce the localization error and select which beacon node the mobile target is closest to in the given area.

A minimum of three beacon nodes are needed to estimate the target's location. A triangulation method is applied to calculate the final position for each target node.

\section{Alerting}

After calculating the mobile target's position, each mobile target has to send its current coordinates to the coordinator, and then the coordinator must alert beacon nodes which lie near the mobile's target trajectory by sending them "warning" messages so they are aware of the approaching of mobile target nodes and can take proper actions, for instance, activating the beacon nodes in the mobile's target trajectory.

Alerting involves activating appropriate beacon nodes in the network in order to track the target nodes. As soon as any router node $b r_{i}$ receives a high RSS value from the mobile target $M T_{j}$, then the beacon router $b r_{i}$ has to alert the beacon nodes in its range by managing the sleep time for each enddevice node, in order to track the mobile target node.

\section{EXPERIMENT SETUP}

The experimental test-bed consists of 7 beacon nodes and 3 mobile targets. We first made a set of experiments to establish the relationship between RSS measurements and the distance values. The achieved relationship can be used to estimate the distance between beacon and target nodes.

\section{A. Sensor Node Model}

Each sensor node has a unique Medium Access Control (MAC) address and location coordinates. We register the MAC address for each beacon node with its current location and store them in an Access database. This data has to be available to the coordinator, in order to display the position for the mobile target node.

JN5139-EK010 sensor node platform is used in our experiments to evaluate the proposed tracking system. This module offers a low power-consumption, low processor overhead, and low cost platform for WSNs. It supports both complex tree and mesh network topologies providing reliable coverage over large areas.

\section{B. Network Topology}

For evaluation purposes, the proposed localization system was deployed in the FK research area lab $(41.5 \times 11.3 \mathrm{~m})$, and it includes 7 beacon nodes distributed over the FK lab as depicted in Figure 4. 
In our experiments, the sink node is considered as a coordinator and does not participate in the localization process, the only task for it is to receive localization information from mobile nodes and transmit this information to a laptop to display the current positions for each mobile target node.

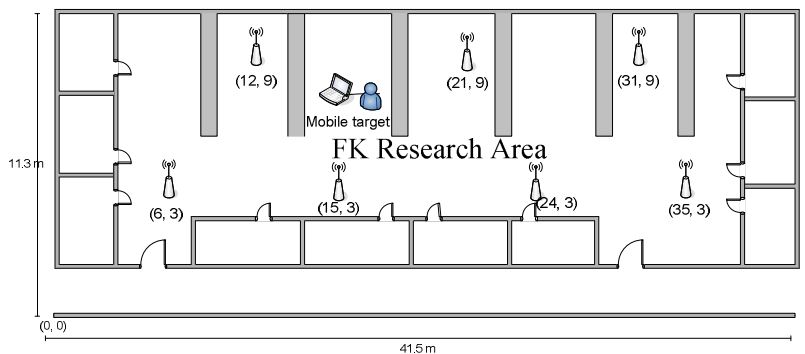

Figure 4. General office test layout

\section{Mobile Target Model}

The mobile target node device is the same as the beacon nodes. The mobile target node has the ability to calculate its current location based on the collected readings from the beacon nodes in its range, and transfer its current location to a coordinator. The mobile target node is connected to a laptop computer in order to display its current position on a map, and its responsible for finding its current coordinates and subsequently transmitting it to a coordinator node every 4-5 seconds.

\section{SYSTEM EVALUATION}

This section reports results obtained through real experiments conducted in indoor environment using Jennic sensor devices. The proposed system was evaluated through measuring the localization error, evaluating the efficiency of tracking multiple mobile targets, and evaluating the interference between sensor nodes.

\section{A. Localization Accuracy}

The localization error $E$ was calculated every 5 seconds based on measuring the difference between the actual position $P\left(\begin{array}{l}x \\ x\end{array}\right)$ and the estimated position $P\left(\begin{array}{l}x \\ x, y\end{array}\right)$ for each mobile node $i$, as presented in Equation (4). The tracking accuracy for Class A, B, and C are evaluated in Figures 5, 6, and 7 respectively.

$$
E=\sqrt{\left(x_{\text {act }}-x_{\text {est }}\right)^{2}+\left(y_{\text {act }}-y_{\text {est }}\right)^{2}}
$$

For simplicity, we propose 3 different localization accuracy classes, and each mobile target has to be assigned to one of these classes. The proposed system will deal with the mobile target nodes as a First Come First Serve principle.

The first case (Class A) includes a single mobile target $M T_{j}$ and 3 beacon nodes $\left(b_{i}, b_{i+1}, b_{i+2}\right)$ in its range. In this case, the mobile target can communicate directly to the three beacon nodes and computes its current position. This class offers the best localization accuracy, as the mobile target node is covered by 3 beacon nodes with known positions.
The second case (Class B) includes a single mobile target $M T_{j}$ with unknown position, another single mobile target $M T_{j+1}$ with known position, and 2 beacon nodes $\left(b_{i}, b_{i+1}\right)$. Through experiments, the tracking accuracy is lower than Class A, as the mobile target node is covered by 2 beacon nodes with known position, and 1 dynamic beacon node.

The third case (Class C) involves a single mobile node with unknown position $M T_{\dot{j}}$, other 2 mobile nodes with known position $\left(M T_{j}, M T_{j+1}\right)$ and 1 beacon router node $b r_{i}$. Class $\mathrm{C}$ offers the worst tracking accuracy as the mobile target nodes is covered by 1 beacon node and 2 dynamic beacon nodes. Table II shows the tracking accuracy and localization error for each class.

TABLE II

LOCALIZATION ACCURACY FOR EACH CLASS

\begin{tabular}{|c|c|c|c|c|}
\hline $\begin{array}{c}\text { Class } \\
\text { Grade }\end{array}$ & $\begin{array}{c}\text { Fo. } \\
\text { Beacon } \\
\text { Nodes }\end{array}$ & $\begin{array}{c}\text { No. Mobile } \\
\text { (Dynamic } \\
\text { Position) }\end{array}$ & $\begin{array}{c}\text { Accuracy } \\
(\%)\end{array}$ & $\begin{array}{c}\text { Localization } \\
\text { Error }(\mathrm{m})\end{array}$ \\
\hline Class A & 3 & 0 & $70-85$ & $0.2-1.5$ \\
\hline Class B & 2 & 1 & $50-65$ & $1-2$ \\
\hline Class C & 1 & 2 & $40-50$ & $1-3$ \\
\hline
\end{tabular}

\section{B. Collision Avoidance}

In order to validate the interference effect, two experiments were conducted; the first one involves transmitting beacon messages from beacon nodes at the same time. The second experiment works by managing transmitting messages to beacon nodes. Figure 8 shows the localization error in both cases.

\section{DISCUSSION}

In this paper, we investigate the ability of tracking multiple mobile targets using ZigBee, in order to reduce the powerconsumption for the whole ZigBee network. The presented system is deployed in indoors environment where GPS, ultrasound, and infrared technologies are inefficient.

Several localization and tracking techniques have been proposed recently for several applications. The implementation of previous studies was limited in scale, as the proposed system in [10], which was implemented in a small area $(300 \times 300 \mathrm{~cm})$, and the proposed system in [7] was implemented where the density of nodes was 0.27 nodes $/ m^{2}$. The proposed work in this paper was implemented in a realistic environment where the density of beacon nodes is low $\left(0.05\right.$ node $\left./ m^{2}\right)$.

ZigBee network standard can achieve a low powerconsumption when the density of end-device nodes is greater than density of router nodes, as the end-devices nodes can go to a hibernation mode as soon as they have completed the required task. The implemented works in [8, 12, 13] concentrate on tracking and localizing a single target node in indoor environment. The mobile target was considered a coordinator in their experiments, which limits the proposed solutions from tracking multiple mobile targets. The proposed system in this paper considers the mobile target as a 
router node in order to track multiple targets nodes synchronously.

The system implemented in this paper does not require strict time synchronization, nor any need for coordination between the beacon nodes, and depends only on the received signal values with no need for any additional hardware. The proposed described in $[9,3,5]$ offer reasonable positioning accuracy but each one requires additional device to be attached to every single node.

\section{CONCLUSION AND FUTURE WORK}

In this paper, we design and implement a tracking system which is able to work with ZigBee wherever the density of router nodes is low. The proposed system is scalable to work in a very large distributed network of sensor nodes. The implemented system has overcome some of tracking limitations such as localization error, communication cost, interference, and the ability to track multiple mobile targets simultaneously. The proposed tracking system was implemented using ZigBee sensor nodes in order to test its validity.

One of the most important developments and improvements that should be added to research in the future is to improve the localization accuracy for the proposed tracking system, by adopting other localization techniques. The tracking system should be deployed in a large area that could include a whole large building composed of several floors. This is important to test the accuracy and efficiency for the proposed tracking system in a large area.

\section{REFERENCES}

[1] T. Alhmiedat, and S. Yang, "A Survey: Localization and Tracking Mobile Targets through Wireless Sensor Network", PGNet International Conference, ISBN: 1-9025-6016-7, 2007.

[2] N. Bulusu, J. Heidemann, and D. Estrin, "GPS-less low-cost outdoor localization for very small devices", IEEE Personal Communications, Vol. 7, pp.28-34, 2000.

[3] B. Jung, and G.S. Sukhatme, "Tracking multiple moving targets using a camera and laser rangefinder. Institute for Robotics and Intelligent Systems Technical Report IRIS-01-397, University of Southern California, 2001

[4] R.Gupta and S. R. Das, "Tracking Moving Targets in a Smart Sensor Network," in Proceeding of the VTC Fall 2003 Symposium, Vol. 5, pp. 3035-3039, Orlando, Florida, USA, Oct. 2003.

[5] D. Li, K. Wong, Y. Hu, and A. Sayeed, "Detection, classification and tracking of targets in distributed sensor networks", IEEE Signal Processing Magazine, vol. 19, no. 2, pp. 17-29, 2002.

[6] W. Park, and M. Yoon, "The implementation of Indoor Location System to Control ZigBee Home Network". SICE-ICASE, International Joint Conference, pp.2158-2161, ISBN: 89950038-4-7, 2006

[7] M. Sugano, T. Kawzoe, Y. Ohta, and M. Murata, "Indoor Localization System using RSSI Measurement of Wireless Sensor Network based on ZigBee Standard," 2006.

[8] J. Blumenthal, R. Grossmann, F. Golatowski, and D. Timmermann, "Weighted Centroid Localization in ZigBee-based Sensor Networks," IEEE International Symposium on Intelligent Signal Processing, WISP, Madrid, Spain, 2007.

[9] S. Takahashi, J. Wong, M. Miyamae, T. Terada, H. Noma, T. Toriyama, K. Kogure, and S. Nishio, "A ZigBee-based Sensor Node for Tracking People's Locations", ACM International Conference Proceeding Series; Vol. 281, New York, USA, 2008
[10] F. Reichenbach, and D. Timmermann, "Indoor Localization with low complexity in wireless sensor networks", Proceeding of the IEEE (INDIN'06), pp.1018-1023, 2006.

[11] W. Jang, and M. J. Skibniewski, "Wireless Networks-Based Tracking and Monitoring on Project Sites of Construction Materials," Journal of Civil Engineering and Management, VGTU, ISSN 1292-3730, 2007.

[12] T. Alhmiedat, and S. Yang. "A Decentralised Low-power ZigBeebased Localization Approach", 14th International Conference on Automation and Computing, Brunel University, West London, UK, 2008.

[13] T. Alhmiedat, and S. Yang, "A ZigBee-based Mobile Tracking System through Wireless Sensor Networks," International Journal of Advanced Mechatroci Systems, vol. 1, no. 1, pp. 63-70, 2008.

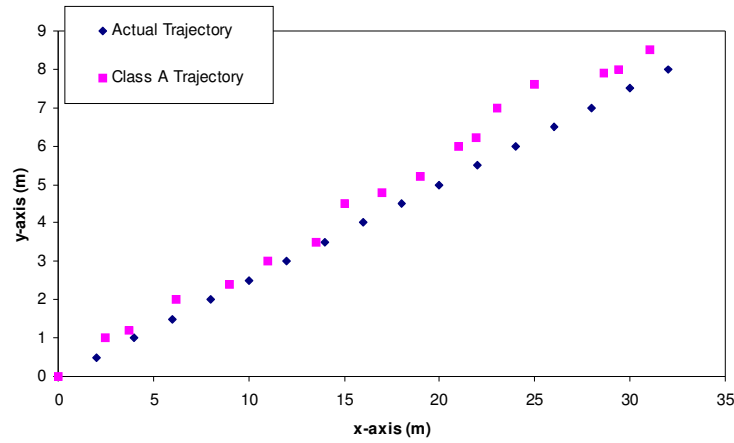

Figure 5. Tracking accuracy for Class A

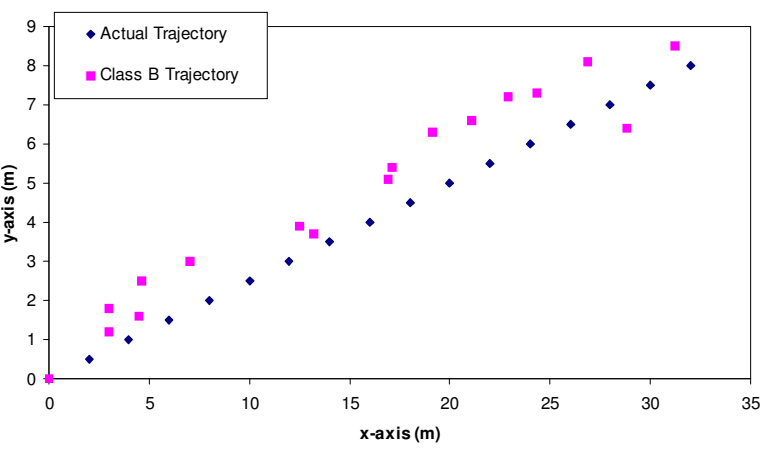

Figure 6. Tracking accuracy for Class B

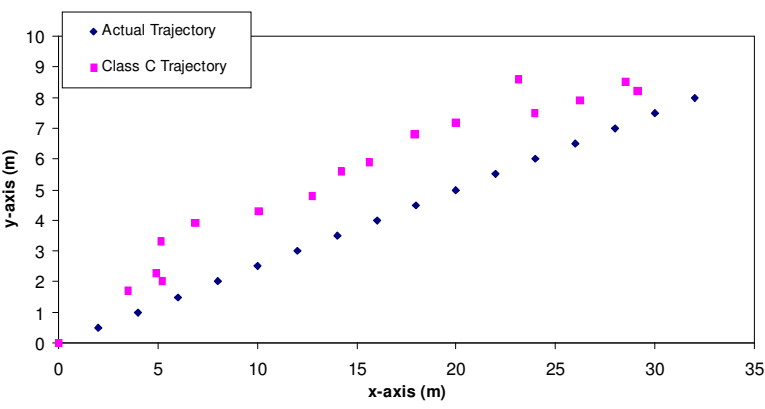

Figure 7. Tracking accuracy for Class $C$

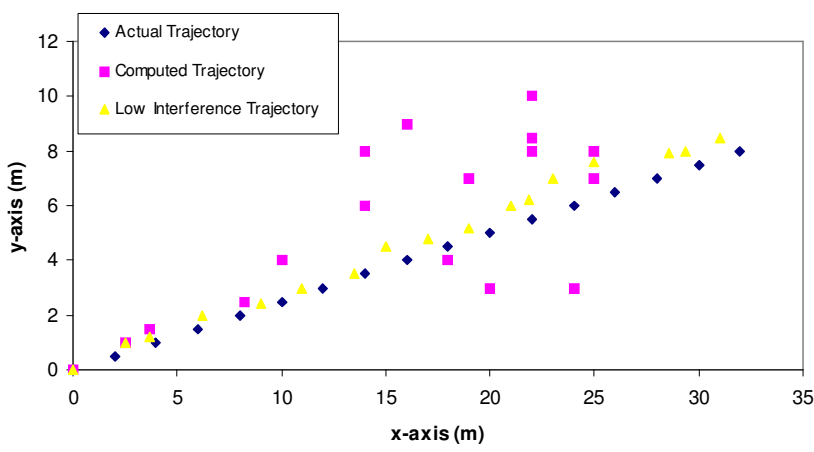

Figure 8. Evaluating the collision avoidance effect 